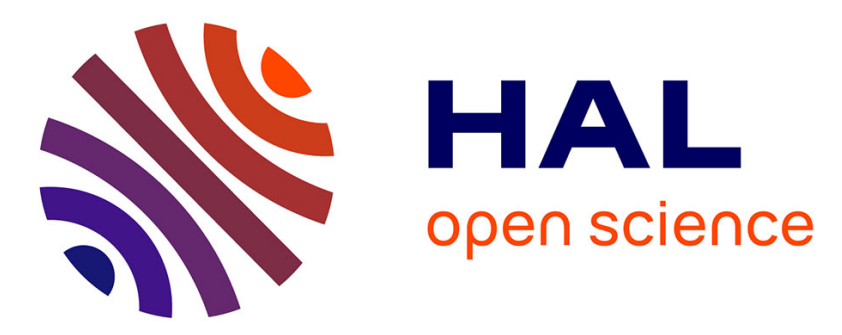

\title{
Dispositif de compensation pour la mise en évidence d'effets oscillatoires de la magnétorésistance en champ magnétique pulsé
}

\author{
Jean Léotin, J.-P. Ulmet, S. Askenazy
}

\section{- To cite this version:}

Jean Léotin, J.-P. Ulmet, S. Askenazy. Dispositif de compensation pour la mise en évidence d'effets oscillatoires de la magnétorésistance en champ magnétique pulsé. Revue de Physique Appliquée, 1969, 4 (4), pp.501-503. 10.1051/rphysap:0196900404050100 。 jpa-00243319

HAL Id: jpa-00243319

https://hal.science/jpa-00243319

Submitted on 1 Jan 1969

HAL is a multi-disciplinary open access archive for the deposit and dissemination of scientific research documents, whether they are published or not. The documents may come from teaching and research institutions in France or abroad, or from public or private research centers.
L'archive ouverte pluridisciplinaire HAL, est destinée au dépôt et à la diffusion de documents scientifiques de niveau recherche, publiés ou non, émanant des établissements d'enseignement et de recherche français ou étrangers, des laboratoires publics ou privés. 


\title{
DISPOSITIF DE GOMPENSATION \\ POUR LA MISE EN ÉVIDENGE D'EFFETS OSGILLATOIRES DE LA MAGNÉTORÉSISTANGE EN GHAMP MAGNÉTIQUE PULSÉ
}

\author{
Par J. LEOTIN, J.-P. ULMET et S. ASKENAZY, \\ Laboratoire de Physique des Solides, associé au C.N.R.S., i I8, route de Narbonne, 3 I-Toulouse. \\ (Reçu le 23 juin 1969, révisé le 22 juillet 1969.)
}

\begin{abstract}
Résumé. - Un dispositif électronique permet d'éliminer la partie monotone de la magnétorésistance au voisinage d'oscillations, pour des mesures effectuées à l'aide d'un champ magnétique pulsé à décroissance exponentielle.
\end{abstract}

Abstract. - A device is described which allows us to keep out the monotonic part of the magnetoresistance near oscillations, when using a pulsed high magnetic field with exponential decay.

1. Introduction. - En présence d'un champ magnétique suffisamment élevé, un conducteur peut présenter des oscillations de la magnétorésistance, riches en information sur la structure de bande : effet Shubnikov-de Haas [1], magnétophonons [2], effet de « spin-splitting » [3], [4].

Des oscillations peuvent être très faibles, par exemple $1 \%$ de la partie monotone, ce qui les rend difficilement observables directement, si la partie monotone n'est pas compensée.

Nous disposons d'un champ magnétique pulsé de $400 \mathrm{kG}$. La nécessité d'éviter les courants de Foucault et les tensions induites sur les sondes de mesure nous a conduits à réaliser un champ de longue durée à décroissance lente [5] réalisée suivant la méthode « crow-bar » [6]. La montée du champ est sinusoïdale. La descente est de la forme $H=H_{0} \exp \left(-\frac{t}{\tau}\right)$ avec une constante de temps $\tau$ de $50 \mathrm{~ms}$ environ (fig. 1).

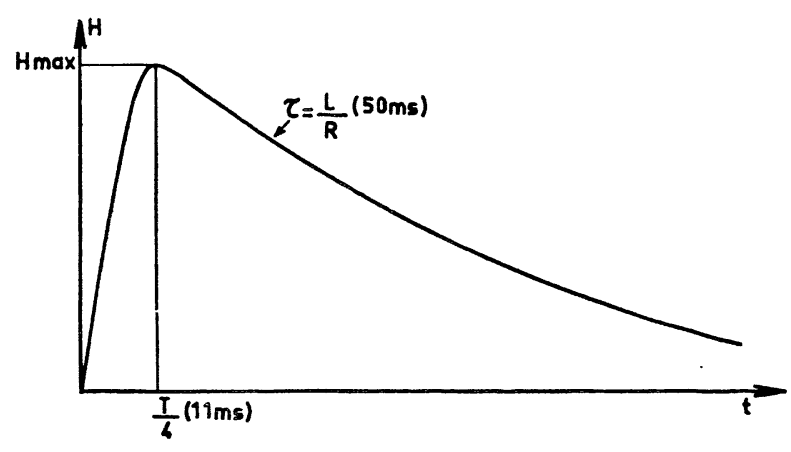

FIG. 1. - Champ magnétique pulsé à décroissance exponentielle.

REVTE DE PHYSIQUe APPLIQUÉE. - T. 4. No 4. DÉCEMBRE 1969.
Nos mesures sont effectuées pendant la descente du champ. L'échantillon est alimenté en courant alternatif constant à la fréquence de $100 \mathrm{kHz}$. La tension $V$ recueillie aux bornes de l'échantillon est proportionnelle à la résistivité $\rho(H)$ de l'échantillon :

L'information est contenue dans une modulation d'amplitude à spectre étroit car la décroissance du champ est lente. La tension aux bornes de l'échantillon est amplifiée sélectivement afin d'éliminer les tensions parasites induites. Après détection, nous obtenons directement sur un oscilloscope à mémoire la courbe $\frac{\Delta \rho(H)}{\rho_{0}}($ fig. 4$)$.

2. Principe de la compensation. - Souvent la magnétorésistance peut être représentée par la relation :

$$
\frac{\Delta \rho(H)}{\rho_{0}}=a H^{\alpha}+f(H)
$$

$a H^{\alpha}$ correspond à la partie monotone et $f(H)$ représente la partie oscillatoire.

Le dispositif que nous avons construit permet de compenser cette partie monotone afin d'isoler et amplifier les oscillations. Comme la décroissance du champ est exponentielle, la partie monotone de la magnétorésistance suit alors une loi exponentielle :

$$
a H^{\alpha}=a H_{0}^{\alpha} \exp \left(-\frac{t}{\frac{\tau}{\alpha}}\right) \text {. }
$$

Pour annuler cette partie monotone, nous opposons à la tension détectée proportionnelle à $\frac{\Delta \rho(H)}{\rho_{0}}$ une 32 

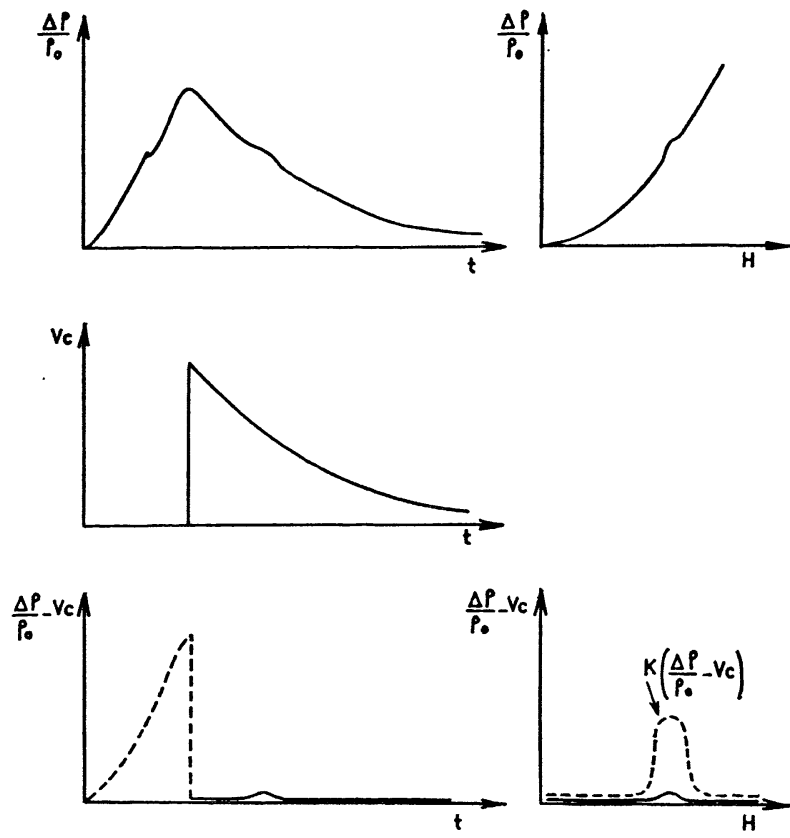

FIG. 2. - Principe de la compensation exponentielle

tension exponentielle $V_{c}$ d'amplitude et de constante de temps convenables (fig. 2).

Si la magnétorésistance ne suit pas une même loi de variation dans sa totalité, il est toujours possible de réaliser une compensation locale, au moins au premier ordre, en faisant coïncider au voisinage d'oscillations les tensions du signal détecté et du signal exponentiel ainsi que leurs tangentes.

Ce type de compensation semble être plus efficace que la compensation réalisée par divers auteurs [7] en opposant un signal proportionnel à $H$ ou en prenant la dérivée seconde en temps de la magnétorésistance.
3. Générateur de tension exponentielle ( $f i g .3)$. Le circuit de base comprend une capacité $C$, chargée sous $15 \mathrm{~V}$. Nous la déchargeons dans une résistance réglable $R$ qui nous permet d'ajuster la constante de temps $\frac{\tau}{\alpha}=R C$.

Cette décharge est commandée au début de la décroissance du champ par un thyristor de faible puissance. Le signal exponentiel est recueilli sur l'émetteur d'un transistor monté en collecteur commun, par l'intermédiaire d'un potentiomètre $P_{1}$ qui règle le niveau de l'exponentielle. Un inverseur permet d'obtenir des signaux positifs ou négatifs suivant le signe de la magnétorésistance. Enfin, grâce au potentiomètre $P_{2}$ et à une pile de $9 \mathrm{~V}$, il est possible de superposer un niveau continu à la tension exponentielle $V_{c}$ afin de compenser la composante continue de la détection.

La différence entre le signal proportionnel à $\frac{\Delta \rho(H)}{\rho_{0}}$ et la tension est directement effectuée et amplifiée au niveau de l'oscilloscope par un tiroir différentiel bas niveau ( fig. 3).

4. Exemple d'application. - Ge dispositif nous a permis de mettre en évidence quatre oscillations de magnétophonons de la magnétorésistance transverse d'un échantillon de GaAs épitaxial de type $n$, dopé à $3 \times 10^{15}$ porteurs [8]. Ces oscillations, dues à la diffusion inélastique des électrons par les phonons optiques longitudinaux, se produisent lorsque l'énergie des phonons est un multiple de l'énergie de Landau, c'est-à-dire $\hbar \omega_{l}=\hbar \frac{e H}{m^{*}} \Delta n$ avec $\Delta n=1,2,3,4 \ldots$

La connaissance de $\omega_{l}$ et des valeurs de $H$ aux résonances permet de calculer $m^{*}$, masse effective des électrons. La figure 4 montre les courbes de magnétorésistance obtenues pour différentes températures de l'échantillon ( $\mathrm{fig} .4 \mathrm{a}$ ), et les oscillations après la

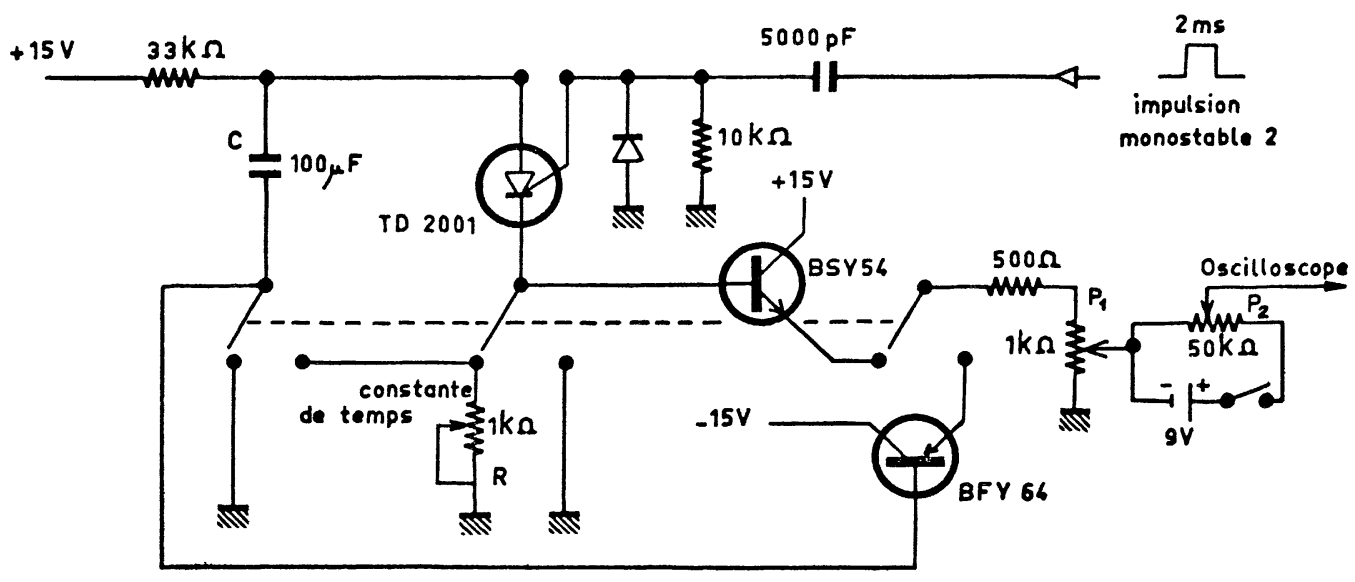

Fig. 3. - Schéma du générateur de tensions exponentielles. 
compensation opérée sur la magnétorésistance à 300 oK (fig. 4 b). Les abscisses des oscillations sont ainsi poin-

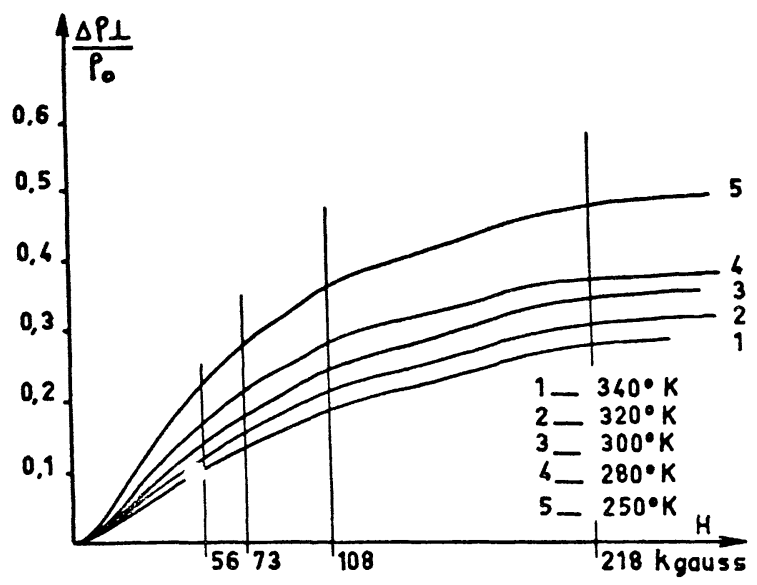

FIG. 4 a. - Magnétorésistance transverse d'un échantillon de GaAs type $n\left(3 \times 10^{15}\right.$ porteurs $)$.

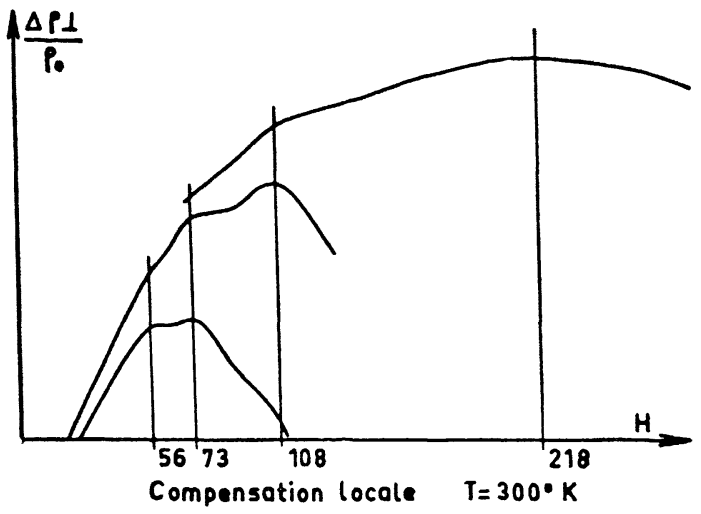

FIG. 4 b.

Magnétorésistance compensée $\left(T=300^{\circ} \mathrm{K}\right)$.

tées avec précision et permettent de déterminer la masse effective des électrons de la bande de conduction du GaAs, soit : $m^{*}=0,0700 \pm 0,0035 m_{0}$.

\section{BIBLIOGRAPHIE}

[1] Lifshitz (I. M.) et KOSEVICH (L. M.), Sov. Phys. JETP, 1958, 6, 67.

[2] Gurevich (V. L.), Firsov (Yu. A.), Parfentev (R. V.) et Shaly' (S. S.), 7e Congrès Semi-cond., Paris, 1964, 6-2, 653.

[3] TSIDILKOVSKI (I. M.), AKSELROD (M. M.) et URITSKy (S. I.), Phys. Stat. Sol., 1965, 12, 667.

[4] Gurevich (L. E.) et Efrros (A. L.), Sov. Phys. $J E T P, 1963, \mathbf{1 6}, 402$.
[5] Askenazy (S.), Santouil (A.) et Cichosz (J.), C. R. Acad. Sci. Paris, 1967, 264, 807, série B.

[6] Levine (M. A.), Combes (L. S.) et GalitaGHer (C. C.), Rev. Scientific Instruments, 1961, 32, 9.

[7] Tsidilkovski (I. M.) et Akseirod (M. M.), Conf. Semi-cond., Kyoto, 1966, IX-2, 362.

[8] Askenazy (S.), Leotin (J.) et Ulmei (J. P.) et al., C. R. Acad. Sci. Paris, 1969, 268, 412, série B. 